\title{
Iterative Multi-Mode Discretization: Applications to Co-Clustering
}

\author{
Hadi Fanaee- $\mathrm{T}^{1[0000-0001-8413-963 X]}$ and Magne Thoresen ${ }^{20000-0003-1511-5938]}$ \\ ${ }^{1}$ Center for Applied Intelligent Systems Research, Halmstad University, Halmstad, Sweden \\ hadi.fanaee@hh.se \\ 2 Department of Biostatistics, University of Oslo, Oslo, Norway \\ magne.thoresen@medisin.uio.no
}

\begin{abstract}
We introduce a new concept called "Iterative Multi-Mode Discretization (IMMD)" which is a new type of efficient data sparsification that can scale up many tasks in data mining. In this paper we demonstrate the application of IMMD in co-clustering, i.e. simultaneous clustering of the rows and columns in a matrix. We propose IMMD-CC, a novel co-clustering algorithm, which is developed based on IMMD. IMMD-CC has attractive properties. First, its time complexity is linear, so it can be used in large-scale problems. In addition, IMMD-CC is able to estimate the number of co-clusters automatically, and more accurate than state-of-the-art methods. We demonstrate the performance of IMMD-CC in comparison to several state-of-the-art methods on 100 data sets from a benchmark cohort, as well as 35 real-world datasets. The results show the promising potential of the proposed method.
\end{abstract}

Keywords: Co-clustering $\cdot$ Bi-clustering $\cdot$ Discretization.

\section{Introduction}

Many real-life datasets are represented by a two-dimensional $n$ by $m$ data matrix, sometimes referred to as two-mode data [5]. The first mode is usually samples (e.g. cases, persons) and the second dimension refers to features (e.g. measurements, genes). It is of great interest in many applications to group rows or columns to meaningful clusters. In the recent years several algorithms are developed for clustering. Recently, motivated by applications in text/web mining, bioinformatics, marketing, and ecology [5] a new version of clustering methods are being developed that simultaneously cluster rows and columns of a data matrix. These methods are usually referred to as co-clustering, biclustering, block clustering, or two-mode clustering. For instance, in analyzing gene expression data, co-clustering can discover functionally related gene sets under different subsets of experimental conditions [18].

The main problem with current co-clustering methods is that many of the them are developed for applications where the volume of data is considered small, so the majority of developed methods are not scalable and are not feasible for very large datasets. Second, the majority of methods, as their clustering counterparts, require the number of clusters as an input, which is much more complicated to set when there are complex 
two-way structures in the data. Besides, since some of these methods have no restriction regarding overlapping co-clusters, they produce an extremely large solution space as the output.

In a very large matrix with billions of rows and columns, where we should look for the co-clusters? Can we somehow limit our search space to more interesting parts of the matrix? Can we find the approximate locations of co-clusters more efficiently (preferably with a linearly in time) and without requiring the user to set the number of co-clusters? These are the questions we attempt to answer in this research. We demonstrate that they can be addressed via Iterative Multi-Mode Discretization (IMMD), a new general concept that we propose. So, our contributions are as follows.

- We propose "Iterative Multi-Mode Discretization" (IMMD), a general and efficient sparsification method for large matrices with real values.

- We propose a new method based on the IMMD concept to extract the matrix's point of interests and then approximate co-clusters without any requirement for the user to specify the number of co-clusters.

- We provide empirical evidences on the promising performance of our method in spotting the known clusters by exhaustive comparison against state-of-the-art methods on tens of benchmark simulated and real-life data sets.

\section{Background}

Suppose that $A$ is an $n \times m$ data matrix, where the elements of this matrix are real numbers and $n$ and $m$ represent the number of samples and features, respectively represented by sets of rows $X$ and columns $Y$. Each element of the matrix $a_{i j}$ represents the relation between row $i$ and column $j$. The matrix $A$ can be defined with its set of rows $X=\left\{x_{1}, . ., x_{n}\right\}$ and columns $Y=\left\{y_{1}, . ., y_{m}\right\}$, so that we can denote $A$ with $(X, Y)$. Also let's denote a set of rows $I \subseteq X$ and columns $J \subseteq Y$, that constitute the submatrix $A_{I J}=\{I, J\}$ that contains only those elements of $a_{i j}$ belonging to the subset of rows $I=\left\{i_{1}, . ., i_{d}\right\} ; d \leq n$ and columns $J=\left\{j_{1}, . ., j_{r}\right\} ; r \leq m$, and vice versa.

Co-clustering is defined as a task of finding a set of elements $C_{k}=\left(I_{k}, J_{k}\right)$, specifically sets of rows $I_{k}$ that exhibit similar behavior across the set of columns $J_{k}$, and vice versa.

Several methods have been developed for co-clustering, which the majority of them are covered in the recent surveys [12], [1], [20], [13], [24].

\section{Co-clustering via Iterative Multi-Mode Discretization}

In this section we introduce the idea of Iterative Multi-Mode Discretization and later show how we extend this idea to co-clustering.

\subsection{Multi-mode discretization}

Continuous data are among the most common data types and can be found in all areas. They can be positive or negative with different units and variances. Hence, normaliza- 
tion or transformation is an essential step in any co-clustering algorithm. Normalization is typically performed column-wise using the following formula.

$$
A_{i, j}^{c}=\frac{a_{i j}-\min _{j}}{\max _{j}-\min _{j}}
$$

Similarly, a row-wise normalization can be performed as follows.

$$
A_{i, j}^{r}=\frac{a_{i j}-\min _{i}}{\max _{i}-\min _{i}}
$$

We can extend Eq. 1 to obtain the discretized matrix $Z^{c}$ with elements $Z_{i, j}^{c}$ between 1 and $s$.

$$
Z^{c}=\operatorname{round}\left((s-1) \times A^{c}\right)+1
$$

Similarly, we can obtain the row-wise discretization as well:

$$
Z^{r}=\operatorname{round}\left((s-1) \times A^{r}\right)+1
$$

Here, $s$ is a scale parameter which should be an odd, natural number $\geq 3$. This multi-mode transformation converts the continuous data matrix $A$ with elements $\in \mathbb{R}$ to two discrete matrices $Z^{r}$ and $Z^{c}$ with elements $\in\{1, \ldots, s\}$.

The benefit of this type of discretization is that similar data points in any row or column will automatically get allocated the same value. So there will be no need to perform the exhaustive search to find groups of similar elements across rows and columns. This is the main and the most important trick. A real-world example of this can be wavelengths in the eye's color perception. Among several lights arriving to our eye we simply focus on one specific wavelength to identify, for example a red object. So, in the co-clustering case, if we want to find the first group of data items, we just need to retrieve the elements whose values are equal to 1 in the transformed matrices.

The reason why we do the transformation across both rows and columns (multimode) is to capture the joint information among samples (rows) and features (columns), in a more efficient way without the need for searching.

\subsection{Iterative removal of non-interesting elements}

The second component of IMMD relies on the removal of non-interesting data elements in an iterative process.

After obtaining $Z^{r}$ and $Z^{c}$ with scale parameter $s$, from these matrices we remove those elements that are not equal to either $\{1, v, s\}$, where $v$ is the median of $\{1, \ldots, s\}$. For instance, if $s=9, v$ will be equal to 5 . So all elements except those that are equal to 1,5 , and 9 are removed. The goal of this stage is to reduce the search space as much as possible by keeping only floor, median, and ceiling values at each iteration. Our assumption is that the co-clusters of interest should appear with more signal power in the final sparse matrices. After the removal phase we generate a new copy of the input matrix that contains only the values of non-removed indices, and for the rest we put missing value (denoted by N/A). We repeat this procedure iteratively, for both modes until we obtain a high-quality sparse discrete matrix for both modes. 


\subsection{Intersection of hotspots across the modes}

After removing phase in an iterative process on both modes and after reaching to the stopping criterion (obtaining an empty matrix), we have to the select the iteration that leads to a better sparsification quality (the statistics we use is described in the algorithm details). In the final matrices we replace those elements that are equal to either $1, v$, or $s$ with 1 and replace the other elements with zero, which leads to a binary matrix respectively for column-wise and row-wise discretization. We then add these two binary matrices, which leads to the intersection matrix. The elements with value of 2 in the intersection matrix represent the matrix's point of interest that appeared as hotspots in both row-wise and column-wise discretization.

\subsection{Finding co-clusters}

Due to the high-level of sparsity in the output matrices, co-clusters normally appear with a reasonable distance from each other and we can identify them by connectivity criteria (a bit similar, but different to algorithms like DBSCAN [4]). We first sort the elements based on the repeated counts of their indices in the remaining items. Then we start from the points whose elements have higher frequent indicies. We create a cluster for the first point. If the second point is reachable (either via row or column) from the first point with a reasonable support it goes to the same cluster, otherwise we create a new cluster for the second point. We continue this procedure until we have no further non-allocated points.

\subsection{Pruning co-clusters}

In this phase we remove those clusters that have a low number of members, lower than a predefined threshold. This is a controlling parameter to avoid overwhelming number of clusters with low number of members.

\subsection{Algorithm IMMD-CC}

Our proposed algorithm IMMD-CC is presented in Algorithm 1. The input of the algorithm are as follows. The input matrix $A(n \times m)$ with continuous real numbers; $\epsilon$, co-cluster's connectivity support threshold; $q$, the minimum number of members for each co-cluster; $h$, the top-h number of hotspots in the mode intersection phase; $s$, the scale parameter for discretization. The output of the algorithm is the co-clusters in the form of indices of rows and columns of the matrix grouped in each cluster.

Note that lines 4-13 and 14-23 are identical. The former is the process based on row-wise normalization and the latter is same process with column-wise normalization. Here we describe the process for the former, but the same explanations can be used for the column-wise part.

We begin with multi-mode discretization as described in section 3.1. We first obtain the discretized version of the matrix $\left(Z^{r}\right)$ in line 7 using the parameter $s$. Then we create a copy of the original matrix A called $I_{i}$ and remove from $I_{i}$ those elements where their corresponding values in $Z^{r}$ is not either 1 (floor), v (median), nor s (ceiling). 
This is done in line 9 . For instance, suppose a $3 \times 2$ matrix which original values are $A=\{0.1,0.1 ; 0.2,0.3 ; 0.3,0.9\}$. Its column-wise discretization transformation is $Z^{c}=\{1,1 ; 3,2 ; 5,5\}$ in the scale of $s=5$. The median of $\{1: s\}=\{1,2,3,4,5\}$ is 3. So, from $A$ we remove those elements that are neither 1,3 nor 5. So the new data becomes $\{0.1,0.1 ; 0.2, N / A ; 0.3,0.9\}$. Then this new data gets replaced with the original data (new $\mathrm{A}$ ) and we repeat this procedure until all values of the matrix becomes $N / A$.

At each iteration we also keep a statistics $O_{i, k}$ (line 12) that allows us chose the best sparsification. Let us denote the number of elements whose values are equal to $1, v$, or $s$ in the matrix $Z^{r}$, respectively with $n_{1}, n_{v}$, and $n_{s}$. Then our statistics is computed as follows. The sum of absolute values of pairwise differences between $n_{1}$, and $n_{v}$ and $n_{s}$ divided by number of available elements in the iteration. For instance, in the above example $n_{1}=1, n_{3}=2, n_{5}=1$. Also the number of available elements is 4 out 5 (only one N/A at the first iteration). So, $O_{i}$ at iteration $k=1$ is computed as $(|1-2|+|1-1|+|2-1|) / 4=2 / 4=0.5$. We keep this statistics at each step, because later at line 24 we want to select the best sparsification based on the lowest obtained value for $O$ over all iterations.

After selecting the best sparsification, the lines after line 26 correspond to the coclustering phase. Based on the selected iteration $k s$ and $p s$ we pick the data output at the end of these iterations. Then we make two temporary matrices $T_{i}$ and $T_{j}$, that are binary (boolean) copy of the data for respectively row-wise and column-wise scenarios. Following the above example, let us suppose that we conclude the first iteration $(k=1)$ gives the best sparsification. Then we have $T_{j}=\{1,1 ; 1,0 ; 1,1\}$, and $T_{i}=\{1,1 ; 1,1 ; 1,1\}$. Since normalization based on row and column give different discretization transformation the values of these are certainly different.

At line 28 we add $T_{i}$ to $T_{j}$ and generate the intersection matrix $T$. Again following the above example our intersection matrix is $T=\{2,2 ; 2,0 ; 2,2\}$. Since both matrices are binary, the values equal to 2 in $T$ demonstrate the intersection of hotspots obtained both row-based and column-based. They are somehow important points of data that depending on the level of sparsification can be exactly the centroid or important members of co-clusters. So, in the next lines (30-31) we obtain the corresponding indices related to these hotspots.

Next, in a procedure that is outlined in lines 32-41 we first generate a new empty matrix $G$ which is the same size as the original data matrix. Then we start from the list of hotspots and add +1 to the corresponding indices of rows and columns of hotspot. If we sort the elements based on their count in the $G$ matrix from higher to lower values we obtain a list of ranked hotspots from the highest important ones to less important items at the end of list.

The first item in the ranked list is with high probability the centroid of the most important cluster. So we create a cluster with this item as its first member (line 42). Then we check the second-ranked item in the list of hotspots. If there is a sufficient connectivity support of this point with the previously allocated element, then it goes to the same cluster, otherwise we create a new cluster and put the new element inside that. The criterion we use for connectivity support is that we count of the number of times that either the row or column index of the candidate has appeared jointly in the 
pair of hotspots. Then we divide this by the maximum obtained repeating count, which gives a value between 0 and 1 . Values close to 0 indicates that the candidate is less connected to the previous member, and values close to 1 means that the candidate is very well connected to the previous element. This procedure continues until all items of hotspots are allocated to a cluster. In order to make the algorithm more efficient we set a parameter $h$ to only do the allocation for top- $h$ hotspots.

Finally, the algorithm ends after a pruning step (line 51) to remove those co-clusters that do not have sufficient number of members. This is controlled with the parameter $q$.

\section{Experimental Evaluation}

\subsection{Datasets}

Simulated datasets The simulated datasets are generated by [16]. We have five groups of 20 datasets, each composed of 500 rows and 200 columns with a constant number of $\mathrm{k}$ co-clusters in each group. Each co-coluster (sub-matrix of $50 \times 50$ ) contains zero values on its elements and the remaining elements of each dataset that do not belong to that co-cluster, are generated i.i.d. from $N(0,1)$.

Real-world datasets The real datasets are publicly available benchmark datasets [22], which consists of 35 microarray datasets related to cancer for different tissue types (eg., blood, lung, colon, breast, skin, prostate, etc.).

\subsection{Compared Methods}

We compare our method with 11 state-of-the-art methods from five categories of techniques: 1) Greedy methods: Cheng and Church (CC) [2], Large Average Submatrices (LAS) [21]; 2) Divide-and-conquer algorithms: Binary Inclusion-Maximal Biclustering Algorithm (BiMax) [19], Qualitative BiClustering (QUBIC) [11]; 3) Distribution parameter identification method: Modified Plaid Algorithm (Plaid) [23], Spectral Biclustering (kSpectral), Bipartite Spectral Graph Partitioning (BSGP) [10], Factor Analysis for Bicluster Acquisition (FABIA) [6]; and 4) Information-theoric algorithms: Information-Theoretic Co-clustering (ITL) [3]; and Fuzzy methods: FuzzyBiClustering [8].

\subsection{Evaluation Metric}

Accuracy Horta and Campello [7] benchmarked 14 quality metrics for co-clustering algorithms and suggested that co-clustering error (CE) proposed by [17] is one of the most appropriate metrics for comparison of co-clustering algorithms. So, we exploit CE as our accuracy metric. Let us denote the found co-cluster with $\mathcal{C}_{1}$ and the reference coclusters with $\mathcal{C}_{2}$. Then let us denote the union of two co-clusterings by $U=\left|C_{1} \cup C_{2}\right|$. Now we can define $\mathrm{CE}$ as:

$$
\mathbb{S}_{c e}=1-\frac{|U|-D_{\max }}{|U|}
$$




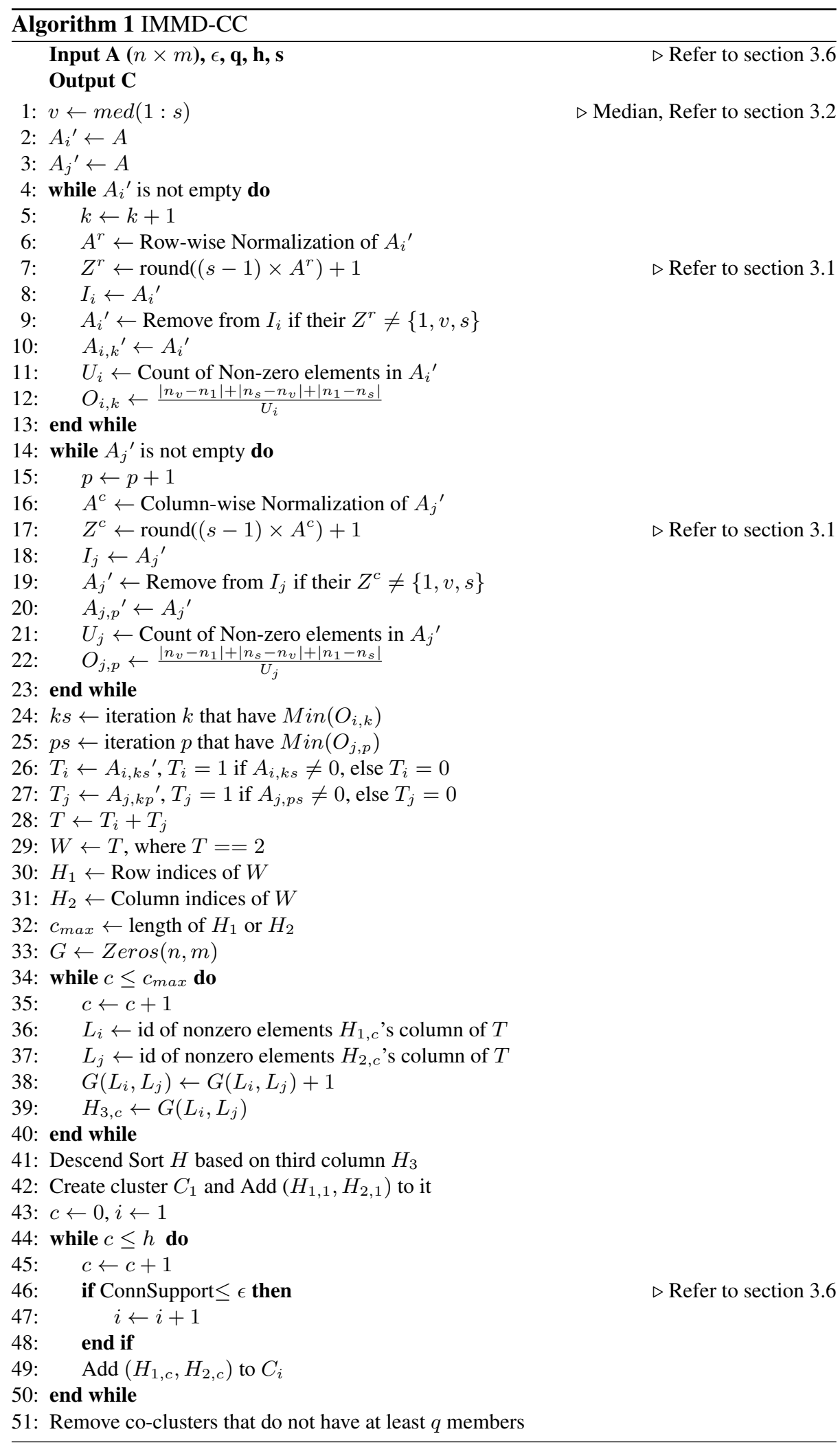


Where $D_{\max }$ is the sum of the diagonal elements of the confusion matrix of $\mathcal{C}_{1}$ and $\mathcal{C}_{2}$ whose elements are the intersection of $\mathcal{C}_{1}$ and $\mathcal{C}_{2}$, i.e., $m_{i j}=\left|C_{1} \cap C_{2}\right|$.

Estimation of number of clusters Another important factor that needs to be taken into account when choosing a co-clustering method is how accurately the method can identify the true clusters without having prior knowledge about the true number of clusters. We use this as one of our evaluation metrics.

Coverage Co-clustering is a more difficult problem compared to regular clustering algorithms that do not have any restrictions with regard to overlapping co-clusters. So we might have two co-clusters with a large portion of overlap. In order to evaluate this we introduce a measure called Coverage as the following. This metric is relative and can be used only for comparison. It penalizes the methods that produce lots of overlapping co-clusters.

$$
\text { Covg }=\frac{\sum C_{c}}{M}
$$

Where $\sum C_{c}$ represents the total area of found co-clusters and $M$ is total area. Values closer to zero demonstrates compact solution space and less overlapping clusters.

\subsection{Experimental Configurations}

We set the parameters as follows. For our method IMMD-CC we set the following parameters $\epsilon=0.3, q=2, s=9$, and $h=\infty$. For other methods we use the implementations available in the MTBA toolbox [9] (Available publically at http://www.iitk.ac.in/idea/mtba/) with the default parameters suggested by the toolbox. Two methods (kSpectral and FuzzyBiClustering) were excluded on experiments with real-life datasets, due to the some bugs in the implementation, which made them infeasible for some datasets.

\subsection{Results}

In this section we report the results from the analysis of both simulated datasets and real-world datasets.

Simulated datasets The box plot of co-clustering accuracy (measured by CE) for 100 datasets is presented in Fig. 1. The surprising observation is that among 11 compared methods only five has a reasonable performance on the simulated datasets. The reason might be that our simulated datasets include only non-overlapping co-clusters and some of these methods may be designed for particular types of co-clusters with specific patterns (see examples in Fig. 1 of [15]).

As we can see from the results, among the 11 compared methods, only ITL [3] outperforms IMMD-CC. However, when we look at the number of times where IMMD-CC identifies exactly the correct number of simulated co-clusters with zero error tolerance ( $k=\{1,2,3,4,5\})$ with respect to the ground truth, IMMD-CC stands out by a large 
difference beyond the other methods, including ITL. In 35 out of 100 datasets, IMMD$\mathrm{CC}$ finds the exact number of simulated co-clusters, while the second-ranked method, CC [2] and third-ranked method, Qubic [11] find the correct number in 20, and 17 respectively of the datasets.

Real-life datasets One of the limitations of the studies on co-clustering problems is the lack of availability of datasets with a ground truth. This is already pointed out by [16] in their co-clustering benchmarking study. They used regular clustering datasets to evaluate the performance of co-clustering algorithms. This makes sense because each cluster in a matrix is a special case of a co-cluster, where one mode includes all rows or columns. However, still the real value of co-clustering algorithms that generate submatrices cannot be evaluated via this strategy.

Regarding the simulated datasets, since the ground truth on the exact location of coclusters is available we could directly measure the accuracy of co-clustering using coclustering evaluation metrics. However, regarding the real datasets, since we do not have real co-clusters, maybe using the same measures for co-clustering might not be ideal. Besides, neither of the metrics measure the compactibility of solution space. For this reason, we instead measure the performance of algorithms in estimation of clusters with respect to their output coverage (see section 4.3). In Table 1 we present the estimated number of clusters together with the coverage.

Table 1 should be read as follows. The first column is the true number of clusters chosen by domain experts in different cancer studies. Then we have pairs of columns for each method. The first column of each pair illustrates how many of the clusters were found by each method, and the next column is the Covg value described in section 4.3.

IMMD-CC has identified the true clusters with the tolerance of 1 error on at least 14 out of 35 datasets. Regarding these 14 datasets the average coverage is 0.04 . On the other hand ITL can find the true clusters in 12 datasets with a tolerance of 1 error. However, average coverage for ITL is 43.72 which is almost 1000 times larger than IMMD-CC. This is an evidence on the effectiveness of the IMMD method in highquality sparsification, so that the clusters of interest can be identified from a much more compact subspace, compared to methods like ITL.

\section{Conclusion and future work}

We introduce a new family of co-clustering methods based on a new concept called iterative multi-mode discretization. We demonstrate the effectiveness of the method both on simulated and real-life benchmark datasets. Although in terms of accuracy, methods such as ITL [3] present a better average performance compared to our method, if we consider other factors such as true estimation of co-clusters, as well as coverage, our approach is a more competitive technique. Still there are some issues left for future research. As an example, applying IMMD-CC to higher order tensors is one potential direction. Another direction is tuning of the parameters for performance improvement. For instance, we empirically found the scale parameter $s=9$ good enough for all datasets we experimented. However, still there is no systematic way to choose this parameter for other datasets, which should be investigated further. The more important 


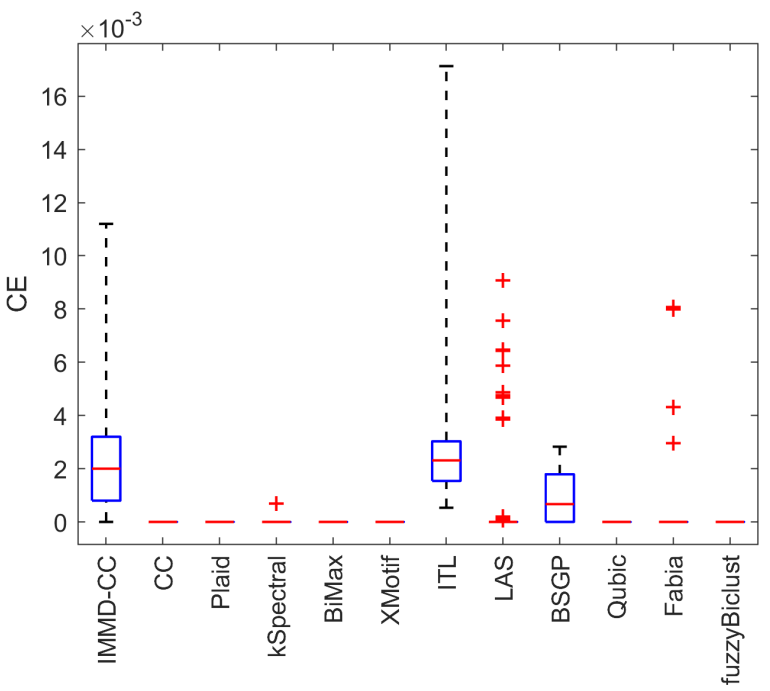

Fig. 1: Co-clustering Quality

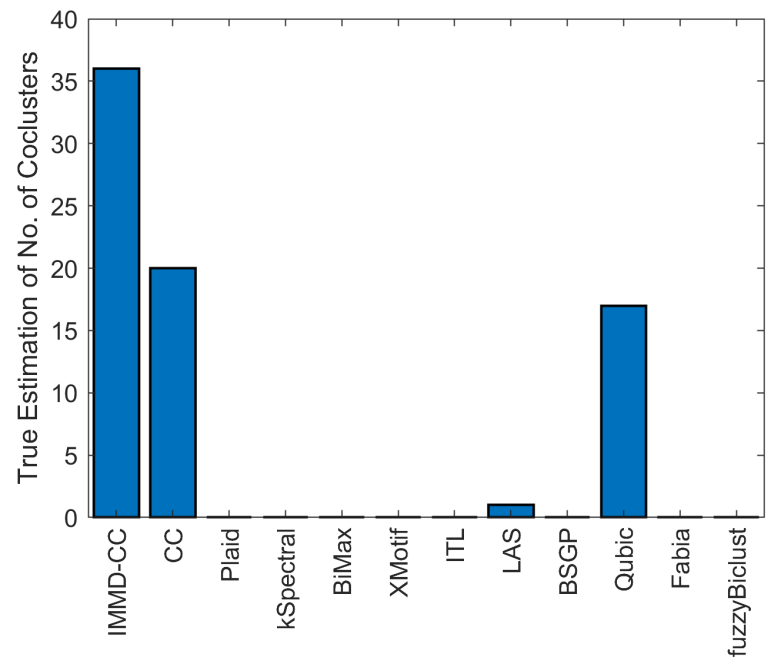

Fig. 2: True estimation of number of co-clusters 
future research should be focused on other applications of IMMC, and not necessarily co-clustering application per se. The IMMC can be considered a highly potential versatile tool in data mining and machine learning. Preliminary work have shown some good results in applications in other problems such as sorting and regular clustering.

\section{References}

1. Charrad, M., Ahmed, M.B.: Simultaneous clustering: A survey. In: International Conference on Pattern Recognition and Machine Intelligence. pp. 370-375. Springer (2011)

2. Cheng, Y., Church, G.M.: Biclustering of expression data. In: Ismb. vol. 8, pp. 93-103 (2000)

3. Dhillon, I.S., Mallela, S., Modha, D.S.: Information-theoretic co-clustering. In: Proceedings of the ninth ACM SIGKDD international conference on Knowledge discovery and data mining. pp. 89-98. ACM (2003)

4. Ester, M., Kriegel, H.P., Sander, J., Xu, X.: A density-based algorithm for discovering clusters a density-based algorithm for discovering clusters in large spatial databases with noise. In: Proceedings of the Second International Conference on Knowledge Discovery and Data Mining. p. 226-231. KDD'96, AAAI Press (1996)

5. Govaert, G., Nadif, M.: Co-clustering: models, algorithms and applications. John Wiley \& Sons (2013)

6. Hochreiter, S., Bodenhofer, U., Heusel, M., Mayr, A., Mitterecker, A., Kasim, A., Khamiakova, T., Van Sanden, S., Lin, D., Talloen, W., et al.: Fabia: factor analysis for bicluster acquisition. Bioinformatics 26(12), 1520-1527 (2010)

7. Horta, D., Campello, R.J.: Similarity measures for comparing biclusterings. IEEE/ACM transactions on computational biology and bioinformatics 11(5), 942-954 (2014)

8. Huang, S.Y., Sun, H.J., Huang, C.D., Chung, I.F., Su, C.H.: A modified fuzzy co-clustering (mfcc) approach for microarray data analysis. In: 2014 IEEE International Conference on Fuzzy Systems (FUZZ-IEEE). pp. 267-272. IEEE (2014)

9. J. K. Gupta, S. Singh, N.K.V.: Mtba: Matlab toolbox for biclustering analysis. pp. 94-97. IEEE (2013)

10. Kluger, Y., Basri, R., Chang, J.T., Gerstein, M.: Spectral biclustering of microarray data: coclustering genes and conditions. Genome research 13(4), 703-716 (2003)

11. Li, G., Ma, Q., Tang, H., Paterson, A.H., Xu, Y.: Qubic: a qualitative biclustering algorithm for analyses of gene expression data. Nucleic acids research 37(15), e101-e101 (2009)

12. Madeira, S.C., Oliveira, A.L.: Biclustering algorithms for biological data analysis: a survey. IEEE/ACM Transactions on Computational Biology and Bioinformatics (TCBB) 1(1), 24 45 (2004)

13. Mounir, M., Hamdy, M.: On biclustering of gene expression data. In: 2015 IEEE Seventh International Conference on Intelligent Computing and Information Systems (ICICIS). pp. 641-648. IEEE (2015)

14. Nemenyi, P.: Distribution-free multiple comparisons. In: Biometrics. vol. 18, p. 263. International Biometric Soc 1441 I ST, NW, SUITE 700, WASHINGTON, DC 20005-2210 (1962)

15. Orzechowski, P., Boryczko, K., Moore, J.H.: Scalable biclustering-the future of big data exploration? GigaScience 8(7), giz078 (2019)

16. Padilha, V.A., Campello, R.J.: A systematic comparative evaluation of biclustering techniques. BMC bioinformatics 18(1), 55 (2017)

17. Patrikainen, A., Meila, M.: Comparing subspace clusterings. IEEE Transactions on Knowledge and Data Engineering 18(7), 902-916 (2006)

18. Pontes, B., Giráldez, R., Aguilar-Ruiz, J.S.: Biclustering on expression data: A review. Journal of biomedical informatics 57, 163-180 (2015) 
19. Prelić, A., Bleuler, S., Zimmermann, P., Wille, A., Bühlmann, P., Gruissem, W., Hennig, L., Thiele, L., Zitzler, E.: A systematic comparison and evaluation of biclustering methods for gene expression data. Bioinformatics 22(9), 1122-1129 (2006)

20. Saber, H.B., ELLOUMI, M.: Dna microarray data analysis: A new survey on biclustering. International Journal for Computational Biology (IJCB) 4(1), 21-37 (2015)

21. Shabalin, A.A., Weigman, V.J., Perou, C.M., Nobel, A.B., et al.: Finding large average submatrices in high dimensional data. The Annals of Applied Statistics 3(3), 985-1012 (2009)

22. de Souto, M.C., Costa, I.G., de Araujo, D.S., Ludermir, T.B., Schliep, A.: Clustering cancer gene expression data: a comparative study. BMC bioinformatics 9(1), 497 (2008)

23. Turner, H., Bailey, T., Krzanowski, W.: Improved biclustering of microarray data demonstrated through systematic performance tests. Computational statistics \& data analysis $\mathbf{4 8}(2)$, 235-254 (2005)

24. Xie, J., Ma, A., Fennell, A., Ma, Q., Zhao, J.: It is time to apply biclustering: a comprehensive review of biclustering applications in biological and biomedical data. Briefings in bioinformatics 1, 16 (2018) 